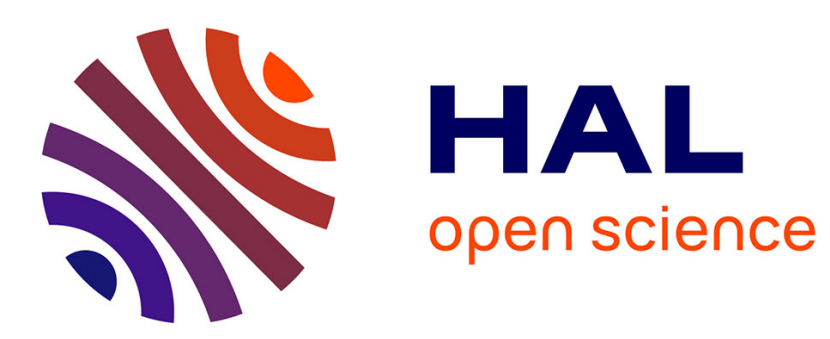

\title{
De l'appel au local comme effet inattendu de l'ubiquité médiatique
}

Francis Jauréguiberry

\section{To cite this version:}

Francis Jauréguiberry. De l'appel au local comme effet inattendu de l'ubiquité médiatique. Espaces et sociétés (Paris, France), 1993, 74-75, pp.117-133. halshs-00680000

\section{HAL Id: halshs-00680000 \\ https://shs.hal.science/halshs-00680000}

Submitted on 16 Mar 2012

HAL is a multi-disciplinary open access archive for the deposit and dissemination of scientific research documents, whether they are published or not. The documents may come from teaching and research institutions in France or abroad, or from public or private research centers.
L'archive ouverte pluridisciplinaire HAL, est destinée au dépôt et à la diffusion de documents scientifiques de niveau recherche, publiés ou non, émanant des établissements d'enseignement et de recherche français ou étrangers, des laboratoires publics ou privés. 
Publié dans la revue Espaces et sociétés, n 74-75, Paris, 1994, pp. 117-132.

\section{De l'appel au local comme effet inattendu de l'ubiquité médiatique}

\section{Francis Jauréguiberry}

On est toujours quelque part, mais de moins en moins là. Ou plus exactement, on est bien toujours quelque part physiquement, mais de moins en moins là autrement. La faculté que chacun porte en soi de s'abstraire de l'ici-présent pour se plonger dans des ailleurs n'est certes pas nouvelle, mais l'actuelle performance des nouvelles technologies de communication permet de la vivre de façon inédite: non plus seulement mentalement (par l'imagination, le rêve ou la construction intellectuelle), mais aussi sensitivement (par le prolongement artificiel de l'ouïe et de la vue) et verbalement (par la voix et l'écrit). De nécessairement successifs et spatialement exclusifs l'un de l'autre, l'ici et l'ailleurs se muent en «possibles » simultanés de réseaux qu'il s'agit d'activer et de gérer.

Jusqu'à l'invention du télégraphe (qui, pour la première fois, a permis à la durée de transmission des informations de s'émanciper de la distance géographique), le contact au monde éloigné était toujours différé, freiné par le temps : temps du voyage, de l'attente du courrier, de l'arrivée des biens et des idées. C'est ce temps, lié au parcours de l'espace, que les outils de télédiffusion (essentiellement la radio puis la télévision) ont relativisé, nous habituant progressivement à l'idée d'ubiquité auditive et visuelle. En zappant, nous pouvons désormais être «transportés » à plusieurs endroits en même temps, et ceci... sans quitter notre fauteuil. Les outils de télécommunication permettent, eux, de concrétiser partiellement cette idée d'ubiquité en rendant possible l'interactivité à distance. Le déterminisme spatial, pour lequel rapidité de l'échange était synonyme de contiguïté a déjà été largement relativisé par l'usage du téléphone : traverser la rue pour parler à un voisin prend plus de temps que composer le numéro de téléphone d'un ami se trouvant à l'autre bout de la Terre. La généralisation, ces dernières années, de la télécopie, de l'EDI (courrier électronique) et des mobiles (téléphones portables) n'a fait que précipiter le mouvement. La commercialisation du visiophone, l'an prochain en France sur le réseau $R N I S$, constituera sans doute (jusqu'à la prochaine invention!) une sorte de point d'arrivée en permettant à la vision interactive de s'émanciper de la proximité physique. La prise de conscience de l'ampleur de ces transformations s'est soldée, à la fin des années 70 et au début des années 80 , par une brusque inflation d'études prospectives destinées à cerner en quoi et comment l'extension de cette 
ubiquité médiatique allait agir sur les modalités de production de nos désormais sociétés communicationnelles.

Après avoir exposé les principaux modes d'approche structurant ces études et rappelé leur contenu, cet article présentera ce que l'on pourrait appeler la «vengeance spatiale » du lien social. Pourquoi «vengeance » ? Parce que presque toutes ces études ont prédit le futur remplacement des espaces territoriaux du lien social par une télésocialité non spatialement définie. Les identifications établies à partir d'un espace physique de référence devaient disparaître au profit d'échanges médiatiques noués autour de thèmes agglutinants. Or, quinze ou vingt ans plus tard, qu'observe-t-on parmi ceux qui sont au plus proche de la situation d'ubiquité médiatique alors imaginée ? Un surprenant (si l'on ne perd pas de vue l'horizon des études prospectives ci-dessus évoquées) appel à ce qui semblait précisément voué à la disparition dans une société de communication : la proximité physique et le local. Seulement cette proximité change de nature, et c'est sur elle qu'il s'agit désormais de s'interroger ${ }^{1}$.

\section{1- Utopies et contre-utopies technicistes : les approches en terme d'impacts}

Une interprétation optimiste des «nouvelles proximités médiatiques » traverse la plupart des études prospectives dont il vient d'être question ${ }^{2}$. Couplées à la microinformatique, les nouvelles technologies de communication y sont décrites dans leur potentialité à rendre possible l'éclatement de la production dans de petites unités à taille humaine dispersées sur tout le territoire avec, comme application extrême, le télétravail à domicile. Reliées entre elles par des réseaux interconnectés hyper performants, ces « unités de production déterritorialisée » laisseraient entrevoir la réalisation d'une décentralisation réussie et d'une autogestion possible ${ }^{3}$. Cette décentralisation permettrait

${ }^{1}$ La démonstration s'appuira sur les conclusions d'une série de cherches menées ces cinq dernières années auprès de gros utilisateurs de terminaux de télécommunication (visiophone, téléphone, radio-téléphone, alphapage et fax). La méthodologie employée a été celle de l'observation participante, d'entretiens individuels et de l'intervention sociologique (réunions de plusieurs groupes d'une dizaine d'usagers chacun durant des séances d'environ deux heures).

2 Voir par exemple Alvin Toffler (Le choc du futur, et La troisième vague, trad. française, Paris, Denoël, 1971 et 1980), relayé en France par Jean-Jacques ServanSchreiber et son Centre mondial pour l'informatique, Joël de Rosnay ou encore Thierry Breton (Sofwar, et Netwar, Paris, Robert Lafont, 1984 et 1987). Le mouvement a institutionnellement été suivi par une série d'appels d'offre émanant de plusieurs ministères afin de prévoir les "retombées sociales et économiques" d'un tel processus.

3 Par exemple : "La société de communication doit être une "société de micro-sociétés" avec des communautés aux dimensions humaines, c'est-à-dire d'une taille moyenne, assez grandes pour traiter en local une majorité de problèmes, mais assez limitées pour que toute la population puisse être rassemblée et embrassée du regard (...). La grande 
à son tour de résoudre les problèmes d'aménagement que l'hypertrophie des métropoles a posés : nuisances (bruit, pollution), baisse de la qualité de la vie (trajet résidencetravail chaque fois plus long et stressant), surcoûts (flambée de la rente foncière, transports en commun déficitaires, frais de gestion urbaine), et lente agonie des campagnes (où vies économiques, culturelles et politiques sont happées par la centralisation urbaine des activités). Par ailleurs, l'agilité des «nouvelles proximités médiatiques » chasserait la pesanteur des relations déterminées par la contiguïté physique et ferait vite oublier le temps perdu avec un voisinage inintéressant ou, selon, la solitude et l'anonymat des grands ensembles résidentiels. Au niveau de l'inscription spatiale de l'organisation sociale, ce serait la fin du modèle pyramidal où le sommet fait figure de centralité toute puissante. À la place, apparaîtrait un système réticulaire sans centre où chaque point de l'espace, en correspondance directe avec l'ensemble des autres, se passerait désormais des intermédiaires qui, jusque-là, jouaient bien souvent un rôle de censeur et d'accaparement que la hiérarchie leur octroyait ${ }^{4}$.

Tous les tenants de cette approche en termes d'impacts ne sont toutefois pas aussi optimistes. Pour certains en effet, l'ubiquité médiatique, loin de permettre aux individus de se rapprocher pour avoir une meilleure prise sur leur destin, risque plutôt de les fixer dans leur solitude en les distrayant pour mieux les exploiter. La critique est ici plutôt culturelle : face à l'adversité, à l'absence de perspective, à la crise ou à l'anomie, il serait possible de fuir, via les nouvelles technologies de communication, vers des «ailleurs» plus en accord avec l'imaginaire et les désirs de chacun. S'économisant les désagréments que toute communication in situ risque de lui faire courir, un individu en mal de reconnaissance ou que le réel désespère peut en effet se

migration urbaine a détruit les communautés, leur unité et leur convivialité, dispersé ou supprimé leurs activités. Les réseaux câblés interactifs, tels les forums d'antan, devraient faire naître ou recréer de nouveaux "villages", même au coeur des zones les plus déshéritées (...). Les réseaux locaux favoriseraient le développement de structures légères et décentralisées ou d'entreprises moyennes (quelques centaines d'employés) que les diverses formes de télétravail permettront d'intégrer progressivement aux nouvelles communautés de vie." (Jean Voge, "La société de communication. Nouveaux médias pour un nouveau monde", Etudes, février 1983, pp. 201-202).

4 "(Dans la société de communication), le radio-concentrisme des échanges et des communications horizontales perd sa valeur au même titre que l'extrême désertification verticale au profit d'une configuration morphologique inapparente où le nodal succède au central dans un environnement électronique prépondérant où la télé-localisation favorise le déploiement d'une excentricité généralisée, périphérie sans fin, signe avant coureur du dépassement de la forme urbaine industrielle". Ce phénomène annonce "la déchéance prochaine des politiques d'aménagement territorial (...), le déclin de l'Etat national $(\ldots)$ et la dérégularisation des différents systèmes d'organisation et de gouvernement (privilégiant la centralité et l'agrégatif)." (Paul Virilio, L'espace critique, Paris, Christian Bourgois, 1984, p. 156 et pp. 115-116). 
brancher sur un réseau ( $C B$, messageries sur minitel, téléconvivialité anonyme, etc.) pour parler de lui, se dire et se représenter tel qu'il voudrait être, en dehors de toute référence sociale et de tout repère d'identification ou code de classement. Seule compte à cet instant l'image qu'il veut bien donner de lui-mêmes.

$\mathrm{Au}$ lieu de freiner la déliquescence du lien social, la désertification des lieux publics de sociabilité et l'enfermement de chacun dans sa sphère privée, les nouvelles technologies de communication ne feraient au contraire qu'accélérer ces phénomènes. Elles précipiteraient l'atomisation des individus en rendant non seulement supportable, mais agréable leur isolement. Elles les installeraient dans une sorte d'hédonisme douillet, les conduisant à s'enfermer chez eux avec un frisson de satisfaction au moment où les discours sécuritaires ne cessent de se développer. Replié sur soi, chacun préférerait désormais consommer ses relations au monde à moindres frais, via des lucarnes électroniques, sans contact direct, sans s'exposer à la critique, au risque ou à l'inconnu, contribuant ainsi à vider un peu plus de toute vie les lieux publics en les rendant par là même encore moins attirants pour ceux qui s'obstineraient à les fréquenter6. Pour les plus pessimistes, on risque de perdre la notion même d'échelle géographique à force de n'entrer en contact avec la réalité qu'à travers des outils qui se jouent des dimensions physiques. À la conjonction de différents terminaux, de plus en

5 Phénomène que Claude Baltz nomme "clivage" dans un article où il analyse les messageries sur minitel ("Messagerie Gretel : images de personne(s)", Réseaux $\mathrm{n}^{\circ}$ 6, 1984). On peut alors se poser la question de savoir si ce que certains cherchent prioritairement à travers les nouvelles technologies de communication n'est pas plus leur auto-valorisation narcissique que le développement des échanges. C'est en tout cas ce que constate Dominique Boullier dans son étude sur les cibistes: "Bien loin de vouloir multiplier les rencontres pour elles-mêmes, les cibistes y cherchent surtout la réhabilitation de leur capacité à s'auto-définir, à s'approprier leur monde, à s'inventer un statut. Ils fuient en quelque sorte une situation sociale où ils se font posséder, où ils ne s'appartiennent plus." ("Vol au dessus d'une bande de citoyens", Réseaux n 20, 1986, p. 44). Michel Bonetti et Jean-Paul Simon vont dans le même sens lorsqu'ils écrivent : "Ce qui est recherché, c'est plus la possibilité d'expression, d'énonciation et à travers ça la reconnaissance par l'autre, qu'on ne cherche pas nécessairement à identifier. Communication narcissique avant tout, où l'on attend du média qu'il permette de s'exprimer, ou bien qu'il parle de soi (fonction miroir), pouvant ainsi contribuer à la réinsertion ou à la valorisation d'individus ou de groupes." ("Les transformations urbaines", Réseaux n² 20, 1986, p. 17).

6 Pour Richard Sennett par exemple, "les communications électroniques sont l'un des moyens par lesquels la notion de vie publique a été étouffée". Certes, la masse d'information qui nous parvient est chaque jour plus importante, "on voit davantage, mais on agit moins ensemble" : c'est le "paradoxe de l'isolement et de la visibilité", paradoxe annonçant le déclin de l'urbanité, des jeux de rôles, des règles de convenance, de la res publica. (Les Tyrannies de l'intimité, trad. française, Paris, Seuil, 1979, pp. 220-221). 
plus dégagé d'un vécu anthropologique de l'espace, l'individu deviendrait totalement dépendant des outils médiatiques dans ses perceptions géographiques, la moindre défaillance de ces outils entraînant des catastrophes existentielles.

Mais, que les descriptions de la future société de communication soient paradisiaques ou apocalyptiques, la démarche qui les produit relève au fond d'une même application mécanique de la pensée de Marshall Mc Luhan : medium is message. Ce qui est important, ce n'est pas le message pris en lui-même (le contenu et le méta-message), mais le mode de communication, c'est-à-dire la technique médiatique employée. C'est elle qui est censée influer directement ou subliminalement sur le socioculturel, voire sur le politique ${ }^{7}$. Le rôle du sociologue, du psychosociologue ou du géographe consiste alors à mesurer les «effets» et les «impacts» des nouvelles technologies de communication sur l'organisation et le changement social ou sur les pratiques de l'espace $^{8}$. C'est contre ce type d'approche techniciste que certains se sont insurgés en rappelant le poids de la reproduction sociale dans l'adoption des technologies.

\section{2- Réponses sociologistes : hiérarchie des espaces et reproduction du social}

Il ne s'agit plus ici, comme précédemment, de partir des technologies pour voir comment elles agissent sur le social, mais du social pour déterminer en quoi sa reproduction conditionne leur développement. Or, force est de reconnaître que la structuration spatiale de notre société est pour l'essentiel inégale, hiérarchique et centralisée. D'où la critique virulente que fait par exemple Jean-Pierre Garnier des visions par trop optimistes évoquées plus haut : "Les réseaux électroniques où circule l'information sont organisés selon une structure hiérarchique et centralisée qui ne fait que redoubler et reproduire, et donc consolider, dans l'espace médiatique, la hiérarchisation et la centralisation propre à la structure de l'espace social, qu'il soit envisagé sous l'angle institutionnel, économique, politique ou culturel (...); il serait illusoire de compter sur l'extension et la densification du réseau médiatique pour mettre fin à la ségrégation sociospatiale (...). Dans une société divisée en classes, la médiation croissante de la communication n'atténue ni, à plus forte raison, n'élimine la hiérarchisation des espaces $»^{9}$.

\footnotetext{
${ }^{7}$ Pour comprendre les média, trad. française, Paris, Mame et Seuil, 1968.

${ }^{8}$ La majeure partie des études livrées en réponse aux appels d'offre ministériels dont il est question en note 1 adoptent cette démarche (voir par exemple les nombreuses "études d'impacts des nouvelles technologies de communication" produites par l'IDATE au début des années 80).

9 "L'espace médiatique : un nouveau lieu pour l'imaginaire social?", in F. Auriac et R. Brunet (dir.), Espaces, jeux et enjeux, Paris, Fayard-Fondation Diderot, 1986, pp. 117118.
} 
La même démonstration est faite pour l'organisation du travail : directement reliés au centre, les acteurs périphériques s'y référeraient chaque fois plus afin d'éviter les conséquences négatives d'une décision inadéquate. Ils s'habitueraient ainsi à une perte d'autonomie de décision et contribueraient par là même au renforcement du modèle pyramidal du pouvoir : «L'accélération des communications a eu pour effet une plus grande centralisation des pouvoirs, une moins grande délégation accordée aux intermédiaires politiques ou commerciaux, quand bien même toute technologie nouvelle en ce domaine permettrait théoriquement le contraire $»^{10}$.

C'est à partir d'une hypothèse semblable que Gérard Claisse organise sa réflexion sur l'espace urbain : «Avant de modifier l'organisation de l'espace, le développement des télécommunications s'inscrit dans une structure spatiale qui conditionne l'architecture et la répartition géographique des réseaux $»^{11}$. Ainsi, est-il démontré, les nouveaux réseaux de communication les plus perfectionnés sont construits, dans leur quasi-totalité, dans les zones de concentration démographique, ce qui accentue encore plus les disparités spatiales. Seules les zones rentables (peu de travaux en génie civil pour un maximum d'abonnés) sont câblées. À l'intérieur des ces zones, une seconde ségrégation intervient alors: les services offerts étant relativement chers, seule une clientèle solvable y a accès.

Plaçant aussi leurs études dans une logique de la reproduction, d'autres auteurs ne contestent pourtant pas que les technologies puissent avoir quelques effets sur le social. Mais, contrairement aux «technicistes », ils pensent que, loin de dynamiquement transformer la société, les nouvelles technologies de communication ne font que la figer dans la reproduction de ses inégalités. Alors que, chez les «technicistes » la technique est présentée comme origine du changement, elle est ici pensée comme condition de la reproduction de l'existant. De telles études renvoient indirectement à l'École critique pour laquelle les médias sont avant tout des outils d'aliénation ${ }^{12}$. En permettant aux habitants des concentrations urbaines, atomes perdus au milieu d'inconnus, de communiquer avec quelques autres atomes connus, mais géographiquement éloignés ou avec des ailleurs purement ludiques ou imaginaires, les nouvelles technologies de communication ne feraient que rendre «supportable » une inscription sociospatiale que tout, par ailleurs, devrait porter à refuser. «La suburbanisation, la périubanisation auraient-elles été "urbainement" possibles sans le téléphone ? Que l'on se souvienne des symptômes du "mal des grands ensembles" dans les années 1960! "Maladies" de déracinement, de migration, de déportation hors des quartiers anciens où la

10 Paul Beaud, La société de connivence, Paris, Aubier, 1984, p. 159.

11 "L'espace et son double", Réseaux n² 20, 1986, p. 54.

12 "Le plus sûr moyen de garrotter la conscience" selon l'expression de Theodor Adorno. 
communication était établie de longue date par le voisinage, mais aussi maladie de l'inorganisation, de l'absence d'équipements de première nécessité (médecin, pharmaciens...), de commerce, de transports. Le téléphone n'est-il pas arrivé à point ? $»^{13}$ De la même façon, la télédistribution, le minitel ou bientôt le visiophone, en permettant de créer un espace convivial artificiel capable de se substituer au moins en partie à un environnement physique peu agréable ou même dévalorisant, désamorceraient d'éventuelles mobilisations visant le changement.

L'ensemble de ces réactions, rappelant l'importance de l'organisation sociospatiale antérieure à l'introduction des nouvelles technologies de communication, est sans doute nécessaire face aux visions par trop déterministes de l'influence de la technique sur le social. Il est évident qu'aucune technologie, quelle qu'elle soit, ne saurait mécaniquement bouleverser les habitudes, les croyances et règles, les systèmes de valeur et de pouvoir, bref les principaux traits de la reproduction sociale des sociétés d'accueil. La conception même des technologies est largement tributaire de cette reproduction ${ }^{14}$. Mais s'en tenir là, sous prétexte que les représentations sociospatiales évoquées plus haut ne sont que fictions face au poids de la reproduction sociale, reviendrait à s'interdire, d'une part de penser l'innovation technologique comme stimulation du changement social et, d'autre part, d'appréhender les représentations suscitées par cette innovation comme révélateur d'attentes sociales.

\section{3- De la prospective comme révélatrice d'attentes sociales}

Il est aisé, plus de dix ans après, de montrer les faiblesses de telles études prospectives. Certes, les conclusions auxquelles certaines aboutissent sont rétrospectivement assez fantaisistes... On aurait cependant tort de les rejeter globalement sous prétexte que leurs prévisions se sont révélées dans l'ensemble inexactes. Car, au-delà de leurs prévisions, ces études prospectives sont non seulement significatives d'une façon de penser, mais aussi révélatrices des attentes et tensions qui travaillaient le corps social qui les a produites. Par exemple, si l'idée selon laquelle le développement des nouvelles technologies de communication allait permettre la décentralisation de certaines activités économiques ou leur éclosion dans des zones jusqu'alors tenues à l'écart du développement est si fréquemment revenue dans ces scénarios prospectifs, c'est certainement qu'elle rencontrait de réels besoins. Si une redéfinition des frontières entre espace de travail et espace de vie, allant dans le sens

${ }^{13}$ Gabriel Dupuy : "Téléphone pour la ville : l'enjeu urbain des centraux", Métropolis $\mathrm{n}^{\circ}$ 52, p. 34.

14 Voir par exemple la démonstration d'Yves Stourdzé, Pour une poignée d'électrons, Paris, Fayard, 1987. 
d'un décloisonnement des activités et d'une meilleure réalisation personnelle, a suscité tant de projections, ou encore si la réappropriation d'espaces publics et conviviaux a stimulé aussi souvent l'imagination, c'est sans doute que ces thèmes faisaient écho à de véritables attentes.

Que ces attentes aient massivement choisi la célébration de la technique (approches technicistes) pour se muer en prophéties ne doit pas étonner. Elles apparaissent en effet à un moment (années 70) où les croyances et idéologies jusqu'alors pourvoyeuses de sens et d'espérance connaissent un déclin certain. Face aux incertitudes que ces dernières ne comblent plus, la technique apparaît aux yeux de beaucoup comme quelque chose de solide, de sûr, d'à la fois incontournable et réconfortant : elle quitte ainsi imperceptiblement sa condition de pure production humaine pour se charger d'une dimension transcendante. $\mathrm{La}$ spectaculaire reconversion médiatique d'anciens idéologues dans la célébration de l'high-tech au cours des années 1980 est peut-être une des dernières tentatives pour collectivement conjurer (avant que d'y «postmodernistement $»$ tomber) la peur du vide laissé par la disparition des grands récits ${ }^{15}$. Les performances quasi magiques des nouvelles technologies de la communication n'ont certainement fait qu'accentuer le phénomène. Raison de plus pour ne pas prendre au pied de la lettre les représentations qu'elles ont suscitées. Il serait sans doute sociologiquement plus rentable de repérer les milieux sociaux ayant produit ces représentations et de poser la question de savoir pourquoi elles ont suscité autant d'espoir ou de crainte parmi certaines couches de la population.

Tel n'est toutefois pas l'objet de ce qui suit. Car entre temps, de prototypes aux effets phantasmés, les nouvelles technologies de la communication sont devenues des outils réellement utilisés. Les conditions de l'ubiquité médiatique imaginée il y a quelques années sont aujourd'hui réalisées: des milliers de personnes en font quotidiennement l'expérience. Prenant précisément comme objet d'étude le vécu d'individus faisant un usage intensif des nouvelles technologies de communication, j'ai pu, d'une part montrer les limites empiriques de l'ubiquité médiatique et, d'autre part et de façon plus fondamentale, enregistrer combien l'appel à la proximité physique et au local était présent chez ceux dont le mode de vie devait « en principe » les abstraire de toute inscription spatiale.

\section{4- Les limites de l'ubiquité médiatique}

15 Jean-François Lyotard, La condition post-moderne, Paris, Minuit, 1979 ; Yves Barel, La société du vide, Paris, Seuil, 1984 ; Olivier Mongin, La peur du vide, Paris, Seuil, 1991. 
Tant qu'il s'agit d'échanger de pures informations instrumentales et fonctionnelles (une commande, un plan, une précision technique), l'agilité médiatique est une source d'économie remarquable (voir par exemple la télécopie), et l'on se loue de pouvoir échapper aux contraintes dues à l'espace. Notre système économique repose chaque fois plus sur ces échanges médiatiques d'informations. Dans ce type de communication, l'information transmise condense à elle seule le motif de l'appel. Le lien unissant les interlocuteurs relève d'une interdépendance fonctionnelle et formalisée. Le correspondant est appelé à cause de son statut et celui-ci suffit à définir la nature de l'échange. Il s'agit là de communications qu'Habermas qualifierait d'utilitaristes et stratégiques, c'est-à-dire uniquement orientées vers le succès de procédures techniques ou par des actions dont le but est d'adapter les moyens jugés les plus rationnels et efficients par rapport à des fins déterminées ${ }^{16}$. Le recours à tel ou tel moyen technique de transmission se fait alors sans état d'âme, pourvu que ce moyen soit le mieux adapté à la nature des informations échangées.

Il en va bien différemment lorsque la communication est entendue non plus seulement comme simple échange d'informations, mais aussi comme relation renvoyant à une communauté intersubjective. Ici, la communication vise tout autant (et souvent plus) à instituer, confirmer ou réactualiser par l'échange la nature de la relation interpersonnelle liant les interlocuteurs qu'à transmettre de purs constats, données ou énoncés fonctionnels. Le vécu intersubjectif de l'échange est central. Au-delà même de ce qui est dit, c'est ce qui est donné à comprendre de la relation qui importe. C'est pour ce type d'échange que la question du choix entre déplacement physique et ubiquité médiatique se pose avec le plus d'acuité. D'une part parce que tout le monde s'accorde spontanément (et seule cette spontanéité importe ici car c'est elle qui est concrètement vécue) pour signaler que, dans ce type de communication, rien ne remplacera la présence physique pour bien se comprendre et, d'autre part, parce qu'un effet pervers lié au développement des télécommunications est en train de prendre une surprenante importance. Je nomme cet effet «survaleur de la présence physique dans un environnement d'ubiquité médiatique ».

Cette survaleur peut être décrite comme suit : à partir du moment où l'on peut faire passer une information par téléphone, visiophone, télécopie ou téléconférence, le choix du déplacement physique pour délivrer cette même information investit l'échange d'une importance intrinsèque inédite. Selon une logique utilitariste, ce choix a en effet un prix (celui, en temps, argent et stress, du déplacement aller-retour) et des inconvénients (par exemple celui d'être plus difficilement joignable pendant ce temps

16 Jürgen Habermas, Théorie de l'agir communicationnel, trad. française, Paris, Fayard, 1987, tome 1, pp 295-296. 
ou celui de se mettre dans une situation où il est beaucoup plus délicat d'écourter et à plus forte raison de couper l'échange). Dans cette optique, une rencontre face à face revient à signifier à son interlocuteur, et ceci avant même que l'échange proprement dit ne commence, qu'on lui accorde une importance particulière. L'échange est davantage vécu comme un don contre don, comme un élan ouvert, que comme une nécessité instrumentale ayant un prix. Du même coup, la position inverse se dessine en filigrane de toute communication médiatisée : si l'on ne fait «que » téléphoner ou visiophoner à son interlocuteur, celui-ci peut croire qu'il ne vaut pas le déplacement... C'est conscients de ce phénomène que beaucoup se croient dans l'obligation de justifier par des explications de type empirique leur recours à la médiatisation électronique dans les cas où, justement, un déplacement physique aurait pu être effectué. Ce phénomène, encore confusément vécu, mais non moins réel, se traduit entre autres choses par l'équation empiriquement vérifiée : plus il y a de télécommunications et plus les déplacements physiques pour « concrétiser », «préciser » ou « vivre » l'échange sont fréquents ${ }^{17}$.

Les médiatisations électroniques ne remplacent donc totalement les déplacements physiques que lorsque ces derniers sont motivés par une stricte délivrance instrumentale d'informations formalisées. Dans tous les autres cas, elles produisent, à des degrés chaque fois divers, un déficit que seul un déplacement physique peut combler. D'où une conclusion, à première vue paradoxale: plus il y aura de télécommunications, et plus il y aura de déplacements physiques. Mais ces derniers changeront alors de nature. Ils seront motivés de moins en moins par des nécessités d'ordre instrumental (les téléinformations les remplaceront très économiquement dans ce cas) et de plus en plus par le désir de vivre les échanges comme création d'intersubjectivité partagée.

\section{5- Le syndrome du zappeur}

La démonstration qui vient d'être faite sur le choix entre "déplacements » médiatiques ou physiques pourrait être renouvelée pour la mise en correspondance entre certains outils médiatiques et certains types de communication, ou pour le choix du silence, de l'attente et du différé, ou encore pour la hiérarchisation des occupations. Dans chaque cas, un même phénomène central est à l'oeuvre : l'individu «branché », que je qualifie de «multidirectionnel», est toujours plus en nécessité de gérer et de programmer sa communication et, de façon plus générale, sa vie. S'il ne veut pas se

17 Parrallèlement, mais allant dans le même sens, on peut constater qu'une bonne partie des conversations téléphoniques servent à évoquer ou organiser des rencontres physiques. Voir Francis Jauréguiberry, Téléinformation, télécommunication et valeur inédite des déplacements physiques, Pau, CNRS UA 911, juin 1992. 
perdre en conjectures dans une situation d'hyper sollicitation, il n'a d'autre issue que d'effectuer des choix en hiérarchisant ses temps, ses lieux et ses relations selon une démarche quasi productiviste. Ce ne sont pas les nouvelles technologies qui poussent l'individu multidirectionnel à plus d'utilitarisme et rendent son action plus instrumentale. Il n'y a là aucun déterminisme. Seulement l'urgence de gestion dont il vient d'être question arrive précisément à un moment où les grands systèmes pourvoyeurs de sens de l'action sont en crise. Faute d'être mue par une éthique de la conviction, l'action semble se résumer à sa performance, à son efficacité.

Une interprétation, que l'on peut qualifier de purement libérale, de cette situation conduit à penser que les «nouvelles proximités médiatiques » permettent une économie de malheur dans notre rapport au monde. À l'image du zappeur face à son poste de télévision, l'individu multidirectionnel instrumentaliserait ce rapport en choisissant la meilleure ou en tout cas la moins mauvaise des possibilités. Libre sur un marché libre, il se composerait une vie à la carte dont la programmation n'aurait d'autre objet que la satisfaction la plus immédiate de ses désirs ou des fins qu'il se fixerait en fonction d'une approche stratégique de la réalité. Cette vision libérale se décline culturellement dans sa version post-moderne : les opportunités et choix se multipliant, la vie deviendrait une sorte de surfing jouissif et parfois esthétique sur des situations qui, à l'image des vagues, seraient à la fois éphémères et constamment renouvelées dans une sorte de mouvement perpétuel. En regard des idéologies et utopies collectives de ce siècle que trop ont cherché à imposer avec les errements que l'on sait, le caractère éphémère et individuel des passions post-modernes serait le meilleur garant des libertés ${ }^{18}$.

L'hypothèse première de mes travaux auprès de gros utilisateurs de nouvelles technologies de communication reprenait au pied de la lettre cette vision. Or, ces travaux n'ont précisément pas permis de vérifier cette interprétation de la réalité. Constat a été fait que l'expérience d'ubiquité conduit moins, ipso facto, à plus de bonheur qu'à une série de doutes, craintes et remises en question que j'ai globalement désignée par le terme de syndrome du zappeur. Les principaux traits de ce syndrome sont, pêle-mêle, une activité fébrile, la peur de perdre quelque chose, la volonté d'être ici et ailleurs en même temps, la nostalgie de l'imprévu, et la recherche d'une authentique intensité de vie et de sentiments profonds ${ }^{19}$. Schématiquement, on peut dire que le syndrome du zappeur renvoie à la tension difficilement vécue par certains entre une rationalisation instrumentale croissante de la vie (efficacité) et une sorte de déficit

18 Voir Gilles Lipovetsky, L'ère du vide et L'empire de l'éphémère, Paris, Gallimard, 1983 et 1987.

19 Voir Francis Jauréguiberry, Un goût d'ubiquité, Pau, CNRS UA 911, 1989, pp. 55-59. 
en sentiments (affect) révélée par cette trop grande ou trop unique rationalisation. D'un côté la traduction stratégique de l'agir communicationnel («il faut que ça rapporte quelque chose »), et d'un autre côté l'aspiration confuse à " autre chose », à un rapport moins instrumental à la réalité : les autres et les institutions doivent pouvoir être pensés autrement que sur le mode de relations à de simples objets, ressources ou fonctions. Cette tension se solde presque toujours par des tiraillements, une inquiétude, un malaise et parfois même de la souffrance.

\section{6- Du local comme métaphore spatiale de la stabilité}

C'est dans les tentatives de résolution de cette tension qu'apparaît chez beaucoup l'appel au local. L'appel au local dont il est ici question est donc tout à fait spécifique. Il renvoie tout entier au syndrome du zappeur. D'autres conduites, visant elles aussi le réinvestissement du local, se développent actuellement avec beaucoup plus de force, en particulier celle, inquiétante, d'individus à la recherche de racines non sociales (détermination physique) parce qu'incapables de se définir socialement, ou celle, plus classique, de défense de territoires en crise économique ou socioculturelle. La démarche ici exposée est au contraire portée par des individus appartenant tous à des catégories socio-professionnelles privilégiées (ingénieurs, techniciens supérieurs, cadres ou professions libérales) et se définissant spontanément comme parfaitement intégrés à la société. On pourrait presque dire que leur appel au local renvoie non pas à un déficit, mais à une sur-intégration sociale. Leur appel au local est une sorte d'épiphénomène possible du syndrome du zappeur. Face aux tensions engendrées par ce syndrome, d'autres réactions sont possibles, en particulier, chez les cadres supérieurs, celle du workoholic: une hyper activité professionnelle mue le travail en une véritable drogue existentielle ${ }^{20}$.

Chez l'hyper branché, chez l'individu multidirectionnel, le local est souvent imaginé (toujours avec une charge émotive) comme lieu unique (territoire géographiquement restreint: quartier, canton, vallée) censé incarner une forme de vérité, d'authenticité et de sensibilité. Le local est d'emblée présenté dans sa capacité de résister au «tout équivalent territorial» produit par le zapping. Pour l'individu multidirectionnel, vivant l'expérience d'ubiquité et souffrant du syndrome du zappeur, l'appel au local est avant tout un désir de paix, de stabilité et de permanence.

20 Cette fuite en avant peut tragiquement terminer : son entreprise faisant faillite, le workoholic ("l'alcoolique du travail") peut soudainement se retrouver sans horizon d'action, plongé en pleine déréliction. On enregistre par ailleurs un nombre croissant de cadres qui "craquent" brusquement (burn out) sans raison particulière et qui refusent ("infantilement" disent ceux qui restent) de revenir à l'environnement de stress permanent qui était le leur. 
Contrairement à ce que pensent les post-modernes optimistes, la conscience de l'aléatoire et de l'éphémère n'est pas toujours grisante et jouissive : elle donne aussi le vertige et la chute n'est pas exclue. Dans un monde où tout s'éparpille, se fragmente et s'accélère, l'individu multidirectionnel, placé en état d'urgence permanent, hésite. Dans son doute, il cherche toujours un élément stable sur lequel il puisse s'appuyer pour se construire en tant que personne. Pour lui, le local peut jouer ce rôle. Il faut y lire comme une réaction à la forme de société informationnelle que Jacques Attali, même s'il ne la souhaite pas, prévoit : une société où «l'homme, comme l'objet, sera nomade, sans adresse ni famille stable, porteur sur lui, en lui, de tout ce qui fera sa valeur sociale » et où «l'éphémère sera le rythme de la loi (et) le narcissisme la source majeure du désir. ${ }^{21}$ Le local fantasmé est alors construit comme le contraire des lieux zappés. Les lieux zappés sont ceux de l'urgence, synonymes des présents successifs sur lesquels s'ouvrent quotidiennement nos agendas. À l'opposé, l'expérience du local renvoie à la permanence, à ce qui fut avant et sera après soi : ce ne sont pas les lieux qui sont éphémères, mais ceux qui y passent. Ici, l'aune de la réflexion n'est pas l'heure, le jour ou la semaine, mais la vie ou, en tout cas, une grande partie de celle-ci22.

Les lieux fréquentés (terminaux des moyens de transport, bureaux, hôtels, foires, etc.) par ceux qui se déplacent physiquement «afin de préciser ou concrétiser ce qu'échangé précédemment par téléphone ou par fax» (les limites de l'ubiquité médiatique...) sont de plus en plus interchangeables. Dans le souci de les rendre le plus praticable possible (effort d'adaptation minimum pour ceux qui y passent), ces lieux finissent par tous se ressembler. Une sorte d'équivalence universelle, dictée par un pragmatisme fonctionnel, est partout vérifiable. La seule particularité de ces lieux consiste en fait dans l'oblitération de toute référence aux espaces culturels qui les englobent. Le local fantasmé est au contraire unique et incomparable. C'est un lieu qui respire et qui vit dans son originalité. Il renvoie à la sensibilité et non à l'efficacité, à l'appartenance et non à l'errance.

Face à la dispersion que le «tourbillon informationnel» peut engendrer, à l'aléatoire trop souvent côtoyé, à l'éphémère renouvelé dans une sorte d'éternel présent, un désir de permanence apparaît. Le local est alors, selon les cas, pensé comme « refuge », transcendance identitaire ou métaphore spatiale de la stabilité. Ce n'est pas le

${ }^{21}$ Lignes d'horizon, Paris, Fayard, 1990, p. 50.

22 Approfondissant cette remarque, on pourrait se demander si l'accélération des présents successifs, trop souvent présentée comme une domination du temps, ne serait pas plutôt une façon d'exhorciser la mort ou, pour le moins, de fuir certaines interrogations d'ordre philosophique. 
« village mondial » cher à Marshall Mc Luhan qui est ici recherché, mais bien plutôt le monde du village, c'est-à-dire, en définitive, celui de l'interconnaissance.

Cette distinction entre mouvement et permanence peut paraître classique. Elle semble renvoyer à ce sur quoi nombre de sociologues du début du siècle ont travaillé : le passage de la communauté à la société (Ferdinand Tönnies) avec la nostalgie de solidarité mécanique (Émile Durkheim) que cela entraînait. Mais la situation vécue par l'homme multidirectionnel n'est plus du tout celle que partageaient ceux qui passaient des communautés villageoises à la ville, de la tradition à la modernité, du toujours déjà connu à l'inconnu. Ils sont au contraire les meilleurs représentants de la victoire moderniste. Tout l'intérêt de la mise en évidence du syndrome du zappeur est de montrer que cette victoire ne peut être totale : c'est chez ceux qui sont le plus près de la réalisation totale de la modernité par le modernisme qu'apparaît ce désir d'exister autrement. J'insère ici ce constat dans la critique qu'Alain Touraine vient de faire de la modernité : celle-ci ne saurait être confondue avec la rationalisation. Elle est aussi subjectivation, c'est-à-dire apparition de la figure du Sujet dans sa liberté23.

\section{7- L'aporie de l'authenticité locale}

Rien ne permet pour l'instant d'imaginer les retombées d'un tel appel au local. Mais, pour ne pas se dérober au thème de la prospective, j'avancerai deux pistes possibles. La première conduit à une situation que je n'appelle pas de mes voeux : la traduction par l'individu multidirectionnel de son appel au local en une action visant à projeter en un seul lieu toute sa subjectivité laissée pour compte par son activité de zappeur. Ce lieu serait pour lui une sorte d'île d'authenticité où il pourrait laisser libre cours à sa spontanéité, à son affect et même à ses penchants d'irrationalité. Une telle conception insulaire du local a de quoi effrayer. Sa réalisation signifierait en effet la dissociation réussie entre, d'une part un lieu central de subjectivité, où le seul mode de communication serait la communion : avec la nature, avec son «être profond ", avec sa famille ou ses égaux (la communauté) et, d'autre part, des lieux éclatés d'instrumentalité vécus comme espaces d'affrontements civilisés entre intérêts particuliers.

23 "Ceux qui veulent identifier la modernité à la seule rationalisation ne parlent du Sujet que pour le réduire à la raison elle-même et pour imposer la dépersonnalisation, le sacrifice de soi et l'identification à l'ordre impersonnel de la nature ou de l'histoire. Le monde moderne est au contraire de plus en plus rempli par la référence à un Sujet qui est liberté, c'est-à-dire qui pose comme principe du bien le contrôle que l'individu exerce sur ses actions et sa situation, et qui lui permet de concevoir et de sentir ses comportements comme des composantes de son histoire personnelle de vie, de se concevoir lui-même comme acteur. Le Sujet est la volonté d'un individu d'agir et d'être reconnu comme acteur." Alain Touraine, Critique de la modernité, Paris, Fayard, 1992, p. 242. 
L'actuelle sectorisation spatiale de grandes métropoles selon les appartenances ethniques, les niveaux de vie ou les échelles de goûts s'accommoderait parfaitement d'un tel néo-communautarisme doublé d'un utilitarisme cynique. Les zappeurs les plus aisés auraient toute chance d'habiter les quartiers chics, voilà tout. On peut toutefois entretenir quelque espoir quant à la non-réalisation d'une telle perspective. Celle-ci impliquerait en effet que l'individu multidirectionnel trouve entièrement son compte affectif dans le vécu d'un tel local insularisé. Or l'expérience de celui-ci risque fort de lui faire apparaître l'écart entre le local désiré (auquel il est demandé d'être authentique et unique) et le local réel (qui est toujours un mélange de particularisme et d'universalisme) : la réification de l'authenticité ne peut que décevoir ${ }^{24}$. La prise de conscience de cet écart pourrait alors s'avérer positive car elle permettrait de rompre la dichotomisation trop bien pensée entre vie privée, locale, affective et authentique d'un côté et vie publique, zappée, rationnelle et instrumentale de l'autre.

La seconde piste conduit à la possibilité d'une nouvelle solidarité locale. Alors que la mobilité a tendance à produire un désengagement, un refus de participer aux choses locales autrement que sur le mode éphémère de la consommation («nous usons les lieux et nous nous en débarrassons tout comme nous jetons kleenex et canettes de bière » écrit Alvin Toffler ${ }^{25}$ ), l'appel au local pourrait se traduire par un attachement producteur d'une réelle implication. Se sentir affectivement, poétiquement, avec émotion, bref subjectivement lié à un territoire, définit une situation en quelque sorte asociale, mais vitale sur laquelle l'acteur peut s'ancrer pour penser le social qui s'y déroule autrement que de façon rentabiliste. Se sentir être ainsi d'un territoire, c'est la possibilité de s'en considérer responsable, ce qui veut dire aussi à l'écoute et solidaire de ceux qui y vivent.

On voit que cette seconde piste va dans une direction opposée à celle de la première. Il ne s'agit pas de revenir au local pour y vivre un enfermement communautaire concomitant d'une utilisation rentabiliste de réseaux universels, mais de vivre le local comme élément de renforcement de la personnalité permettant à l'individu multidirectionnel de se concevoir comme acteur. Ce qui renvoie en définitive à la figure du sujet capable de penser (vivre, revendiquer) sa particularité face au système tout en

${ }^{24}$ En amont, la recherche de l'authenticité se heurte à la mise en scène de plus en plus répandue de cette même authenticité par des villes ou collectivités territoriales voulant associer leur nom à une image de qualité de vie. A terme, leurs stratégies de communication se soldent par une mise en équivalence de lieux qui, pour les acteurs qui nous intéressent ici, ne devraient précisément pas avoir d'équivalents mais être incomparables (il en va de même pour les destinations "exotiques et uniques" des voyagistes).

${ }^{25}$ Le choc du futur, op. cit., p. 90. 
étant un acteur de ce système. Quoi qu'il en soit, il faut s'attendre à ce que le local, loin d'être évacué par l'ubiquité médiatique et le développement des transports physiques, se mue au contraire en un élément incontournable de la pratique et de l'analyse sociales. 